Check for updates

Cite this: RSC Adv., 2019, 9, 12218

\title{
The effect of PEG functionalization on the in vivo behavior and toxicity of CdTe quantum dots $\uparrow$
}

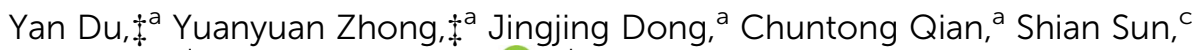 \\ Liping Gao and Dongzhi Yang (D)*ab
}

CdTe quantum dots (QDs) are considered a potential toxic substance because they contain metal ions. However, most toxicology data are derived from in vitro studies or limited in vivo analysis and may not reflect in vivo responses and biodistribution. Proper modification is one of the most widely used routes to reduce the toxicity of QDs. Herein, we demonstrated the role of polyethylene glycol (PEG) in decreasing the toxicity of QDs by studying the animal survival, clinical biochemistry, organ histology, biodistribution and oxidative stress in thioglycolic acid (TGA)- and mercapto-acetohydrazide (TGH)stabilized CdTe QD (TGA/TGH-CdTe QD)-treated groups. Via the histology, transmission electron microscopy (TEM) and biodistribution results, it was found that the QDs mainly accumulated in the liver and kidney at 7 days post-injection, and obvious tissue damage was also observed in the bare TGA/TGHCdTe QD group. Based on the evaluation of oxidative stress in the liver and kidney, the indicators exhibited an obvious variation with a high dose of TGA/TGH-CdTe QDs. In contrast, the QD aggregation decreased in the liver and kidney with no clear physiological index variation after PEG functionalization. Thus, PEG plays an important role in decreasing the toxicity of the CdTe QDs, and both the accumulation of cadmium and oxidative stress variation instead of an isolation factor are responsible for the in vivo toxicity of these QDs.

Received 2nd January 2019

Accepted 2nd April 2019

DOI: 10.1039/c9ra00022d

rsc.li/rsc-advances

\section{Introduction}

Quantum dots (QDs) have shown significant potential in biological and medical applications because of their excellent optical properties. ${ }^{1,2}$ However, Cd and Te quantum dots are considered to be toxic due to their possible release from nanosystems; $;^{3,4}$ moreover, the presence of toxic metal ions, especially the potentially toxic cadmium metal ions, cannot be neglected. Thus, their biomedical utilization has remained a serious biosecurity concern. In recent studies, various new synthetic methods and surface modifications have been used to minimize the surface defects of these QDs and endow them with better biocompatibility. ${ }^{5}$

The QDs studied to date are usually quickly taken up by the liver and spleen, and, to a minor degree, by bone. ${ }^{6,7}$ It has not been shown that whether the QDs leave these organs once

ajiangsu Key Laboratory of New Drug Research and Clinical Pharmacy, Xuzhou Medical University, Xuzhou, Jiangsu 221004, China. E-mail: dongzhiy@xzhmu.edu. cn; Tel: +86-516-63262138

${ }^{b}$ Department of Pharmaceutical Analysis, Xuzhou Medical University, Xuzhou, Jiangsu 221004, China

${ }^{c}$ Xuzhou Air Force College, Xuzhou, Jiangsu 221000, China

${ }^{d}$ Xuzhou Cancer Hospital, Xuzhou, Jiangsu 221000, China

$\dagger$ Electronic supplementary information (ESI) available. See DOI: 10.1039/c9ra00022d

\$ These authors contributed equally to this work. inside or they are ultimately cleared from the organs. Most QDs are made of toxic materials such as cadmium, indium, and arsenide. To reduce the toxicity of QDs, they are usually covered by a nontoxic shell or macromolecules, which serve to protect the toxic part from degradation. Researchers would prefer to use QDs that are eliminated from the body over time rather than those that are essentially deposited in organs forever or until broken down. ${ }^{8-10}$ Thus, it is important to study the in vivo behavior of QDs. However, most toxicity studies have focused on the in vitro assessment and may not provide reference to the biological behavior of these QDs in vivo; this is a significant factor hindering the progress in the clinical application of QDs..1112

The actual application of QDs in biology requires the question about their toxicity in vivo to be addressed. Moreover, the relevance between the dose of exogenous substances and biological reaction can be understood through acute toxicity tests, which can indicate the potential target organs to provide reference for long-term research. ${ }^{13-16}$ In addition, it is highly dependent on the biocompatibility of QDs in biological and biomedical applications. Although QDs can be directly prepared in an aqueous solution without residual organic molecules, their uptake in the reticuloendothelial system (RES) and nonspecific adsorption are not negligible based on previous studies. It was reported that the toxicity of QDs could be reduced by many methods such as by modifying the surface 
area of QDs with polymers or silica encapsulation. ${ }^{17,18}$ Among them, PEGylation (i.e., functionalization by PEG polymers) has been extensively adopted to improve the in vivo behavior and enhance the safety of nanoparticles. ${ }^{19-21}$ It has been reported that the carboxyl groups on the surface of quantum dots lead to nonspecific binding of QDs to oligonucleotides. Thus, methoxyterminated and hydroxyl-terminated PEG coatings play an important role in reducing the nonspecific binding of QDs to cells.

In the present study, PEG was functionalized on the surface of CdTe QDs. By comparing the behavior, weight, organ coefficient, clinical biochemistry, organ histology, biodistribution and oxidative stress of the animal models in the TGA/TGH-CdTe and PEG-TGA/TGH-CdTe QD groups, the effect of PEG on the toxicity of QDs was investigated. The ultramicrostructure and element mapping of the liver and kidney have been further evaluated to determine the accumulation of QDs, which is in accordance with the results of the liver and kidney oxidative stress; this study provides a better understanding of the toxicity mechanism of CdTe QDs at the animal level.

\section{Experimental}

\section{Chemical and reagents}

Tellurium powder (99.999\%) and ethyl thioglycolate were purchased from Jingchun Reagent Co., Ltd (Shanghai, China). Sodium borohydride and cadmium chloride were obtained from Yunzhan Chemical Co., Ltd (Shanghai, China). Hydrazine hydrate $(80 \%)$, polyethylene glycol $4000\left(\mathrm{PEG}_{4 \mathrm{k}}\right)$ and dimethyl sulfoxide (DMSO) were supplied by Xilong Chemical Co., Ltd (Guangdong, China). Absolute ethyl alcohol was obtained from Sinopharm Chemical Reagent Co., Ltd (Shanghai, China). Acetic anhydride was purchased from Shentong Chemical Co., Ltd (Tianjin, China). Colorimetric kits for serum biochemical marker detection were purchased from Nanjing Jiancheng Bioengineering Institute (Nanjing, China). All other materials and reagents were of analytical grade. Ultrapure water $(\geq 18.2$ $\mathrm{M} \Omega$ ) from a Milli-Q system (Millipore, USA) was used in all aqueous solutions.

\section{Preparation of TGA/TGH-CdTe and PEG-TGA/TGH CdTe QDs}

TGA/TGH-CdTe and PEG-TGA/TGH CdTe QDs were synthesized according to our previous procedure. ${ }^{22}$ At first, TGH was prepared via a reaction between ethyl thioglycolate and hydrazine hydrate. Then, TGA/TGH-CdTe QDs were synthesized by the hydrothermal method, in which TGA and TGH with the molar ratio of $1: 6$ were used as stabilizers. Briefly, a $\mathrm{CdCl}_{2}$ aqueous solution $\left(1 \mathrm{mmol} \mathrm{L}^{-1}\right)$ was bubbled with nitrogen for $20 \mathrm{~min}$, followed by the addition of a mixture of TGA and TGH with the $\mathrm{Cd}:(\mathrm{TGA} / \mathrm{TGH})$ molar ratio of $2: 1$. After adjusting the $\mathrm{pH}$ value to 9.0, a freshly prepared NaHTe solution obtained from an oxidation-reduction reaction was swiftly added to the mixture. The solution was allowed to heat at $140{ }^{\circ} \mathrm{C}$ for $60 \mathrm{~min}$ to obtain the TGA/TGH-CdTe QDs, which emitted at $580 \mathrm{~nm}$.

PEG functionalization was carried out by forming a hydrazone chain via a reaction between aldehyde and hydrazine, which endowed the PEG-TGA/TGH CdTe QDs with $\mathrm{pH}$ sensitivity. At first, the aldehyde on PEG was synthesized according to a reported procedure. ${ }^{23}$ Then, the prepared PEG aldehyde was mixed with TGA/TGH CdTe QDs with the weight ratio of $2: 1$ and allowed to react under stirring in the dark at room temperature for $12 \mathrm{~h}$. Both the TGA/TGH CdTe and PEG-TGA/ TGH CdTe QDs were precipitated in acetone ( $\mathrm{v}: \mathrm{v}=1: 3)$ to obtain the purified powder and redissolved in related solutions before use. Both the concentrations of TGA/TGH CdTe QDs and PEG-TGA/TGH CdTe QDs were calculated based on the weight of TGA/TGH CdTe QDs, where the amount of TGA/TGH CdTe QDs in both QDs was kept the same by determining the content of Cd via atomic absorption spectrometry (AAS).

\section{Cytotoxicity evaluation}

Cytotoxicity assessment was conducted in HeLa cells (cervical cancer cells as the tumor cell model). Cells were seeded in a 96well plate for $24 \mathrm{~h}$ before being treated with TGA/TGH-CdTe or PEG-TGA/TGH-CdTe QDs. Both QDs with known concentrations (via serial dilution) were added to the cells. After $24 \mathrm{~h}$ incubation, the relative viability of the cells was determined by a cell titer 96 kit following the manufacturer's protocols. The percentage of viable cells relative to the untreated control was plotted against the QD concentration.

\section{In vivo safety evaluation}

The animal facilities and protocols were approved by the Animal Care and Use Committee of Xuzhou Medical University. All procedures were in accordance with the National Academy of Sciences Guide for the Care and Use of Laboratory Animals. ${ }^{24}$ Equal numbers of male and female Kunming mice in the weight range of 18.0-22.0 $\mathrm{g}$ were obtained from Xuzhou Medical University, which were acclimatized for 7 days before nanoplatform treatment. The mice were housed in standard cages with sawdust bedding, with a controlled $12 \mathrm{~h}$ light/dark cycle. The temperature was maintained at about $20{ }^{\circ} \mathrm{C}$ with a relative humidity of $60 \%$ and an air exchange rate of 15 times per hour. Potable water and standardized diet were provided ad libitum. The high dose group was determined to be $10.0 \mathrm{mg} \mathrm{kg} \mathrm{kg}^{-1}$ according to the results of half lethal dose experiment, and the medium and low dose was calculated based one fifth of the high and medium dose, respectively. Low $\left(0.4 \mathrm{mg} \mathrm{kg}^{-1}\right)$, medium $\left(2 \mathrm{mg} \mathrm{kg}^{-1}\right)$ and high dose $\left(10 \mathrm{mg} \mathrm{kg}^{-1}\right)$ of TGA/TGH-CdTe and PEG-TGA/TGH-CdTe QDs were intravenously (i.v.) injected into healthy mice. The variation in mouse body weight was monitored every other day for 7 days. An approximately $0.5 \mathrm{~mL}$ portion of blood was collected for a mini chemistry panel test before the mouse was euthanatized.

\section{Histology}

Hematoxylin and eosin (H\&E) staining of tissues from the heart, liver, spleen, lung, kidney and genitals (testis/ovary and uterus) was conducted after day $7(n=5)$ and imaged using a light microscope (Olympus BX43F, Japan) to further investigate the potential signs of toxicity (i.e., cellular shrinkage or blebbing, steatosis in liver cells, condensation of chromatin, rupture of 
cell membrane and apoptotic bodies). Transmission electron microscopy (TEM) with element mapping (FEI G2T12, USA) was used to further determine the effect of different QDs on the ultrastructure of the liver and kidney, as well as the aggregation of QDs.

\section{Organ oxidative stress}

Herein, four indicators were measured, i.e. malondialdehyde (MDA), superoxide dismutase (SOD), catalase (CAT) and glutathione peroxidase (GSH-PX), to evaluate the organ oxidative stress. Moreover, $10 \%$ homogenate of liver and kidney was made using a tissue homogenizer (PRO200, USA) under ice bath conditions. Subsequently, the tissue homogenate was centrifuged at the speed of $2000 \mathrm{rpm} \mathrm{min}{ }^{-1}$ at $4{ }^{\circ} \mathrm{C}$ for $10 \mathrm{~min}$, taking the supernatant on standby for oxidative stress measurement following the manufacturer's protocols of Nanjing Jiancheng Bioengineering Institute (Nanjing, China).

\section{Organ distributions}

Organ distribution studies were carried out by AAS to confirm the quantitative tracer uptake values based on the absorption signals obtained from cadmium. After collecting the blood, main organs, including the heart, liver, spleen, lung, kidney and genitals (testis/ovary and uterus), were taken out at 7 days postinjection. After being heated at $500{ }^{\circ} \mathrm{C}$ for $6 \mathrm{~h}$, the samples were dissolved in a $10 \%$ nitric acid solution to determine the organ distributions. The percent of injected dose per gram tissue $(\%$ ID per g) of CdTe QDs in a specific tissue was calculated using the following equation: ${ }^{25}$ was about $3.8 \mathrm{~nm}$, which increased to about $39.6 \mathrm{~nm}$ after functionalization with PEG. The size values obtained via the DLS measurement were higher than those obtained from the TEM images due to the hydration of NPs in an aqueous solution. The optical properties shown in Fig. 1D indicated that both QDs exhibited a continuous absorption profile from 200 to $550 \mathrm{~nm}$ with the characteristic peaks at $536 \mathrm{~nm}$ and $541 \mathrm{~nm}$. Under emission at $350 \mathrm{~nm}$, the maximum emission wavelength of the TGA/TGH-CdTe QDs was $580 \mathrm{~nm}$ and $598 \mathrm{~nm}$ for PEGTGA/TGH-CdTe QDs with an $18 \mathrm{~nm}$ red-shift. Compared with that of Rhodamine $6 \mathrm{~B}$, the quantum efficiency of the TGA/TGHCdTe and PEG-TGA/TGH-CdTe QDs was $27.01 \%$ and $20.86 \%$, respectively.

\section{In vitro toxicity evaluation}

For the cytotoxicity assessment of the TGA/TGH CdTe QDs with and without PEG capping, the effects of TGA/TGH CdTe and PEG-TGA/TGH CdTe QDs at different concentrations on cell viability were investigated in HeLa cells. The results shown in Fig. 2 indicate that the TGA/TGH CdTe QDs exhibited nonnegligible toxicity for HeLa cells, and only $62.2 \%$ of the HeLa cells remained alive after $24 \mathrm{~h}$ incubation with $45 \mu \mathrm{g} \mathrm{mL}{ }^{-1} \mathrm{TGA} /$ TGH CdTe QDs. With an increase in the concentration of QDs, more cells were damaged. At the same concentration of TGA/ TGH CdTe QDs, the cell viability increased after capping of QDs with PEG, and over $87.0 \%$ of the HeLa cells remained alive upon incubation with the PEG-TGA/TGH CdTe QDs. These results revealed that PEG played a role in reducing the cytotoxicity of the QDs.

$$
\% \mathrm{ID} / \mathrm{g}=\frac{([\mathrm{QDs}] \text { in tissue suspension }) \times(\text { volume of tissue suspension })}{([\mathrm{QDs}] \text { in injected solution }) \times(\text { volume of injected } \mathrm{QDs}) \times(\text { wet weight of tissue })}
$$

\section{Statistical analysis}

A minimum sample number of 6 was employed to ensure statistical power. Data was evaluated with analysis of variance (ANOVA), and differences between groups were analyzed for statistical significance using the Bonferroni $t$-test. $p<0.05$ was considered to be statistical difference. $p<0.01$ was considered to be significantly statistical difference.

\section{Results and discussion}

\section{Characterization of the CdTe QDs}

The size and optical properties of the QDs varied with the synthetic conditions. In this study, the QDs synthesized at $140{ }^{\circ} \mathrm{C}$ in $60 \mathrm{~min}$ were used because of their highest quantum efficiency. The morphology and optical properties of the prepared TGA/TGH-CdTe and PEG-TGA/TGH-CdTe QDs were investigated by TEM, UV-Vis absorption and emission spectrometry. As shown in Fig. 1, the average size of TGA/TGH-CdTe

\section{In vivo toxicity evaluation}

The in vivo toxicity of the TGA/TGH CdTe and PEG-TGA/TGH CdTe QDs was also evaluated. Herein, three doses of 0.4 (representing low dose), 2.0 (representing medium dose) and 10.0 (representing high dose) $\mathrm{mg} \mathrm{kg}^{-1}$ QDs based on the weight of the TGA/TGH CdTe QDs were intravenously injected into Kunming mice. The weight variation and living status of the mice were tracked within 7 days. Fig. S1† shows the body weight variation after treatment with different QDs. The results indicated that the QDs affected the body weight of the mice, where the weight growth rate in the high-dose TGA/ TGH CdTe group was obviously lower than that in the control group, and a sleepy and inactive state was also observed after 4 days of injection. Organs were taken out to study the tissue damage, and hepatomegaly of the liver, the main detoxication organ, was clearly observed in the high and mediumdose TGA/TGH CdTe groups. As shown in Fig. 3, the liver coefficients in both groups were significantly higher than 

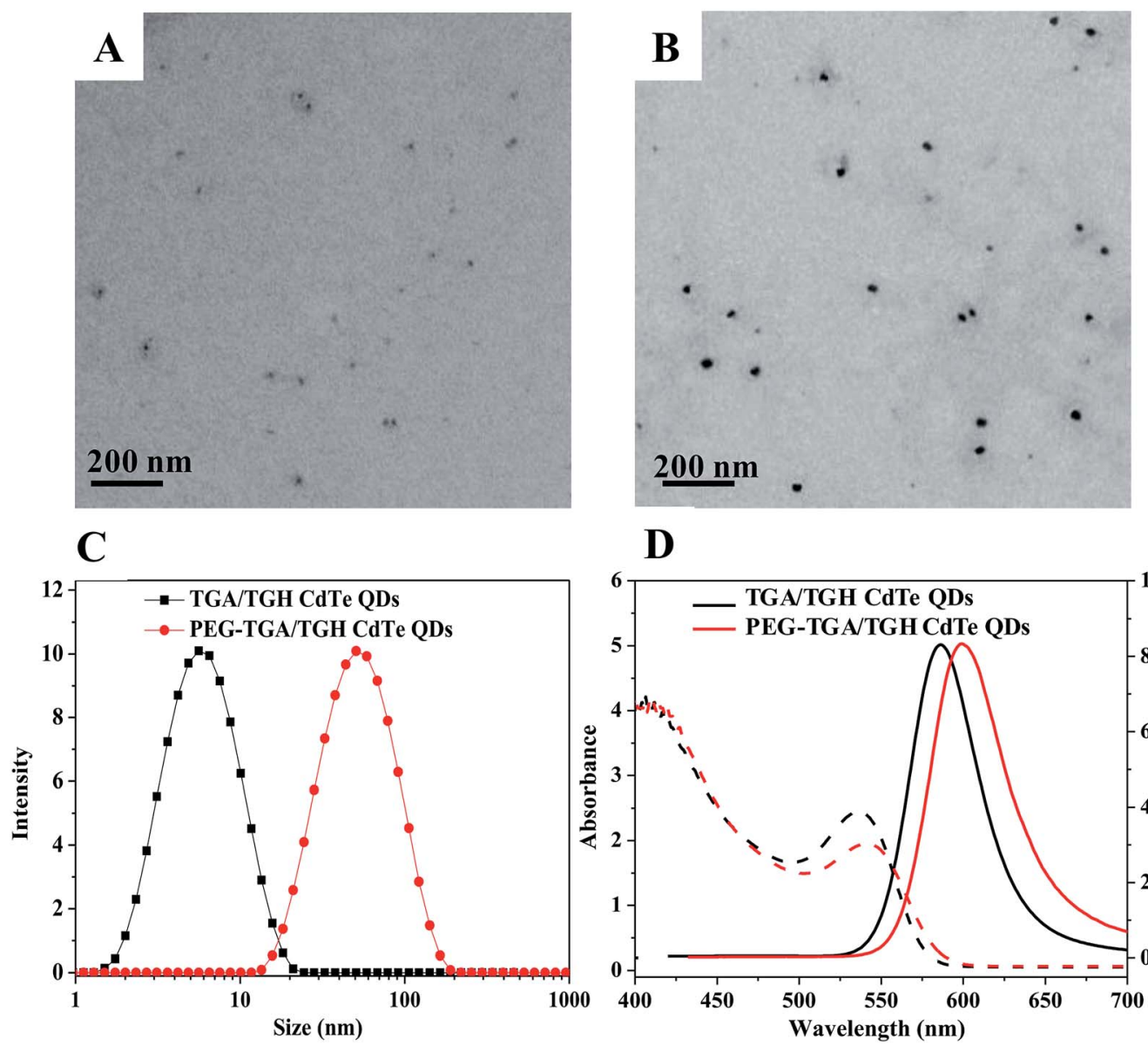

D

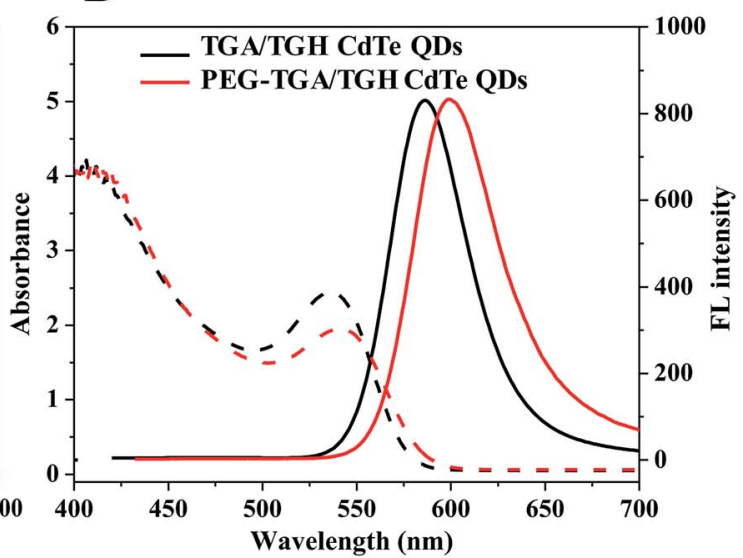

Fig. 1 Structure, particle size distribution and fluorescence spectra of CdTe QDs. (A) TEM image of TGA/TGH CdTe QDs, (B) TEM image of PEGTGA/TGH CdTe QDs, (C) DLS curves of both QDs and (D) optical properties of both QDs.

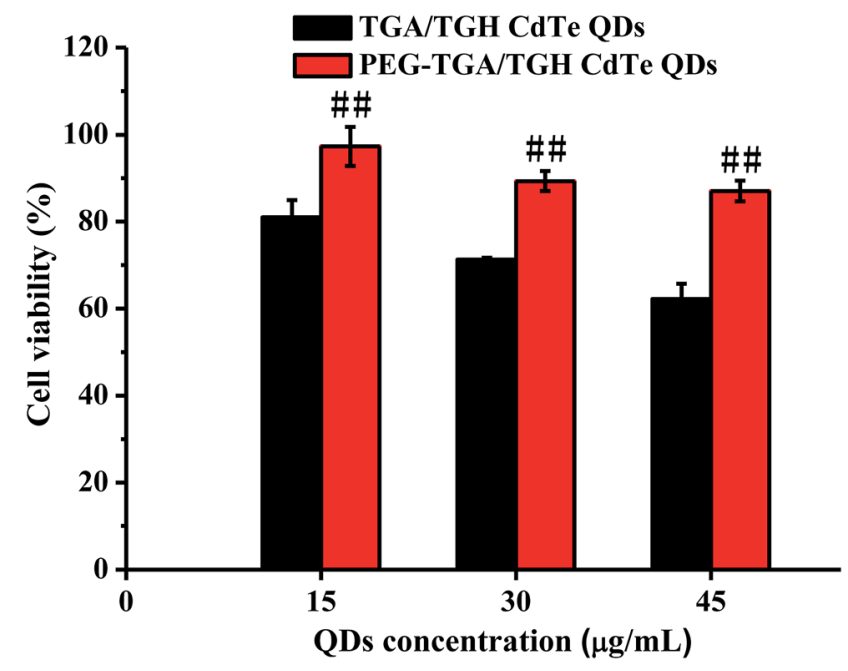

Fig. 2 In vitro cytotoxicity of TGA/TGH CdTe and PEG-TGA/TGH CdTe QDs. ${ }^{\# \#} p<0.01$ vs. TGA/TGH CdTe group.

that in the control group. In contrast, no noticeable weight distinction and side effects were observed in the PEG-TGA/ TGH CdTe QD group.
Histological assessment was carried out on the H\&E-stained slides of the organ (heart, liver, spleen, lung, kidney, testis, ovary and uterus) tissues obtained from sacrificed mice at 7 days post-injection of different QDs. The results shown in Fig. 4 and $2 \mathrm{~S} \uparrow$ indicated that no obvious pathological changes and lesions were observed in the heart, lung, spleen, testis and ovary treated with high dose of TGA/TGH-CdTe or PEG-TGA/TGHCdTe QDs when compared with the case of the control group. However, obvious pathological changes were present in the liver and kidney samples after treatment with high dose of TGA/ TGH-CdTe QDs. The hepatocyte cords, hepatic lobules, hepatocytes, fatty, and nuclei had varying degrees of lesion in the liver. In the kidney, the size of the renal tubular epithelial cells increased, the cytoplasm was stained red, the lumen was irregular, and even cell necrosis appeared. In contrast, no obvious lesions and injuries were observed for all the organs, including the liver and kidney, in the PEG-TGA/TGH-CdTe QD group. These results certified that PEG played an important role in decreasing the toxicity of CdTe QDs.

\section{Ex vivo distribution of QDs}

Generally, cadmium ion leakage and oxidative stress affection, were considered as the main reasons that lead to toxicity of 


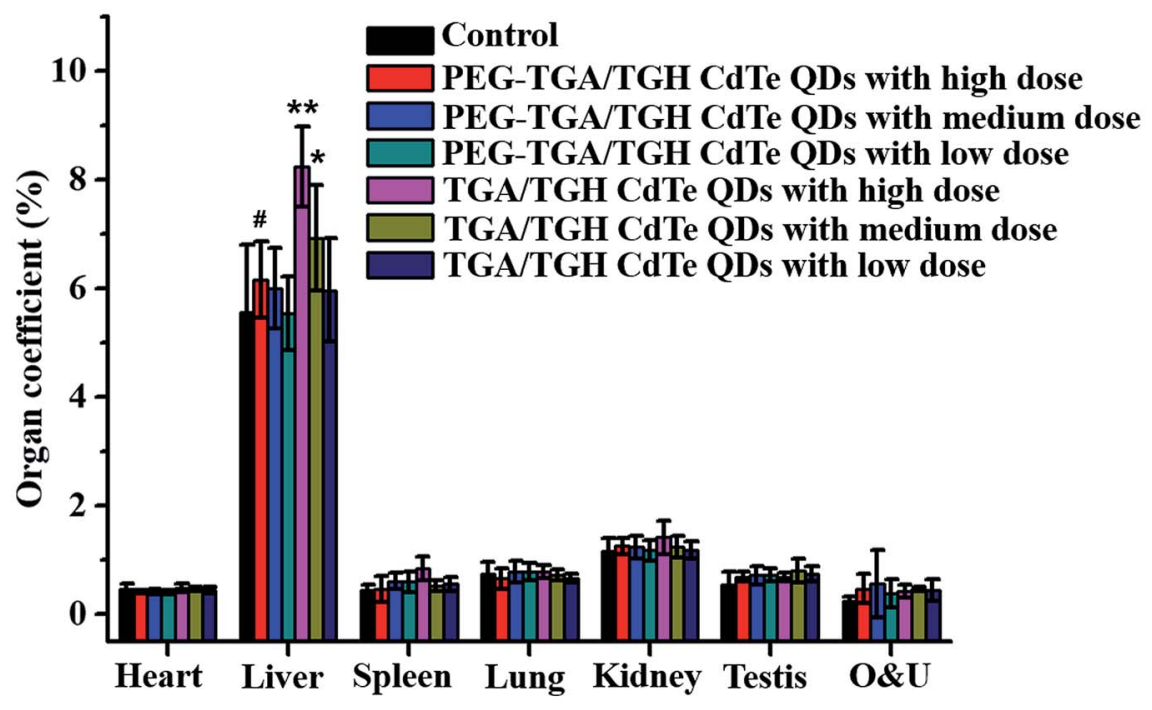

Fig. 3 Organ coefficients of the mice post-treatment with QDs. ${ }^{*} p<0.05$ vs. control group; ${ }^{* *} p<0.01$ vs. control group; ${ }^{*} p<0.05$ vs. TGA/TGH CdTe group.

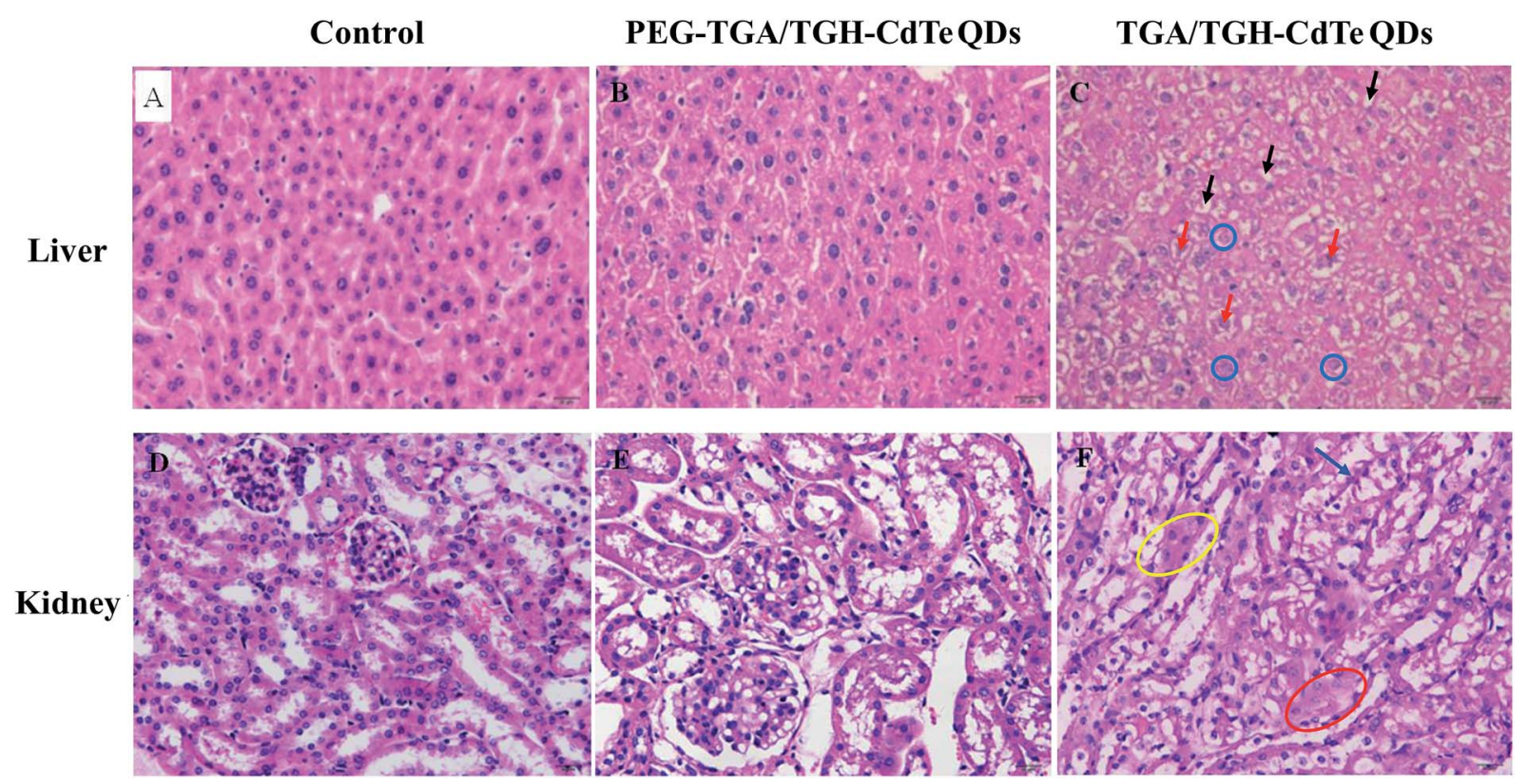

Fig. 4 H\&E staining of the liver and kidney tissues obtained the control group and the mice treated with TGA/TGH-CdTe QDs and PEG-TGA/ TGH-CdTe QDs. Black arrow: fatty degeneration with primarily small bubble fats, red arrow: deformed nucleus, blue circle: destroyed stem cells, blue arrow: irregular renal tubular lumen, yellow elliptic curve: red stained cytoplasm, and red elliptic curve: enlarged tubular epithelial cells.

cadmium-related QDs. Thus, the QD distribution, mini chemistry panel tests and oxidative stress indicators were determined for investigating the mechanism of toxicity of the CdTe QDs.

Cadmium ions can be released when cadmium-containing QDs oxidize; this is responsible for primary liver cell damage. ${ }^{26}$ According to the atomic absorption spectrometry results, as shown in Fig. 5, the amount of CdTe QDs accumulated in the liver and kidney was significantly higher than that in other organs. Furthermore, the concentration of QDs in the liver and kidney was distinctly different for the PEG-TGA/TGH-
CdTe QD and TGA/TGH-CdTe QD groups. Based on the concentration of $\mathrm{Cd}^{2+}, 8.4 \mu \mathrm{g} \mathrm{g}^{-1}$ and $8.8 \mu \mathrm{g} \mathrm{g}^{-1}$ QDs were present in the liver and kidney for the PEG-TGA/TGH-CdTe QD group, and in contrast, $17 \mu \mathrm{g} \mathrm{g}^{-1}$ and $18 \mu \mathrm{g} \mathrm{g}^{-1}$ QDs accumulated in the liver and kidney for the TGA/TGH-CdTe QD group, respectively. Furthermore, the total amount of $\mathrm{Cd}^{2+}$ in the PEGTGA/TGH-CdTe QD group was lower than that in the TGA/TGHCdTe QDs group. This result further confirmed that PEG could relieve the toxicity of QDs by promoting the QDs to be expelled from the body. The liver and kidney were the tissues where the 


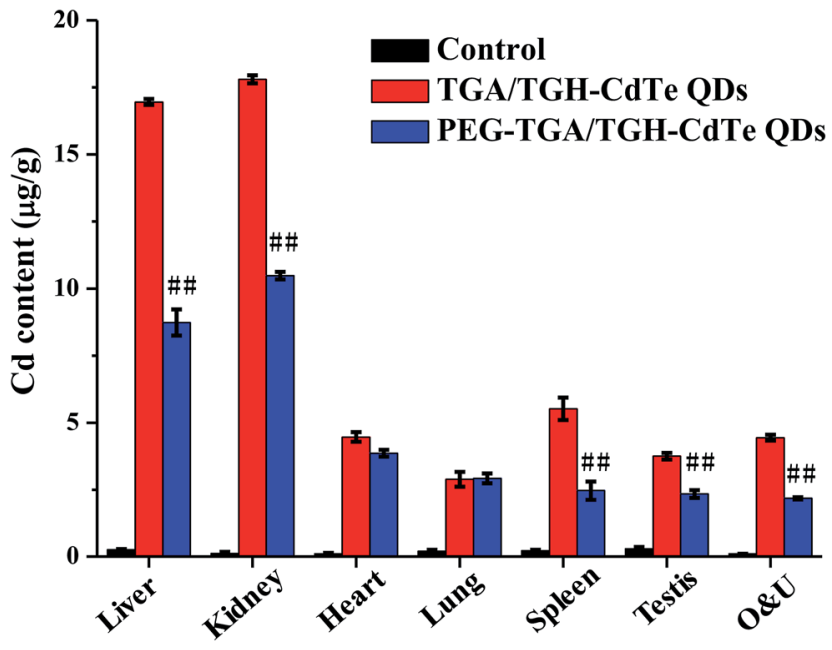

Fig. 5 QD distribution determined by AAs. ${ }^{\# \#} p<0.01$ vs. TGA/TGH CdTe group.

QDs mainly accumulated. Thus, to further study the effect of the QDs on the liver and kidney, their ultramicrostructure was investigated by TEM with element mapping. As shown in Fig. 6, obvious tissue damages were observed in the liver and kidney of the TGA/TGH-CdTe QD group. Hepatocyte swelling with fat drops in the liver cell cytoplasm, nucleus pyknosis, an increase in intracellular lipid droplets, increase in smooth endoplasmic reticulum, cholestasis in the capillary duct, mitochondria deformation and decrease were observed in the liver. A similar phenomenon was observed in the kidney, where the foot process fused, epithelium swelled, mesangial matrix increased, the capillary loops of the glomerulus narrowed and extravasated blood appeared. In contrast, there was no obvious abnormality in the cell ultrastructure of the liver and kidney samples in the control and PEG-TGA/TGH-CdTe QD group. These results were consistent with the H\&E staining observation; this further suggested that the safety of the QDs was increased due to the protection of PEG.

To further study the toxicological impact of the TGA/TGHCdTe QDs and the protective effect of PEG, mini chemistry panel tests including ALB, ALT, AKP, SCr and BUN, which were the indices related to the liver and kidney function, were carried out to determine the general health status of the QD-injected mice. As shown in Table 1, the expression of ALT, SCr, BUN and ALB was significantly changed in the high-dose TGA/TGHCdTe QD group when compared with that in the control group. Inspiringly, all the measured biochemical parameters fell within the normal ranges post-injection of PEG-TGA/TGH-CdTe QDs. Combining the retention information acquired from the distribution via AAS and Cd concentration determined via the TEM results, it may be reasonable to conclude that the PEGTGA/TGH-CdTe QDs will not cause significant toxicity to the test subjects.

\section{Oxidative stress test}

The toxicity of cadmium-containing QDs can be induced by oxidative stress, which is associated with an increase in the production of oxidizing species or a significant decrease in the effectiveness of antioxidant defenses and generated by the nanoparticle itself or by the physical destruction of the cellular activity. ${ }^{27}$ Due to the complexity in vivo, the toxicity of QDs is not easily measured and quantified in animals. ${ }^{28}$ Moreover,

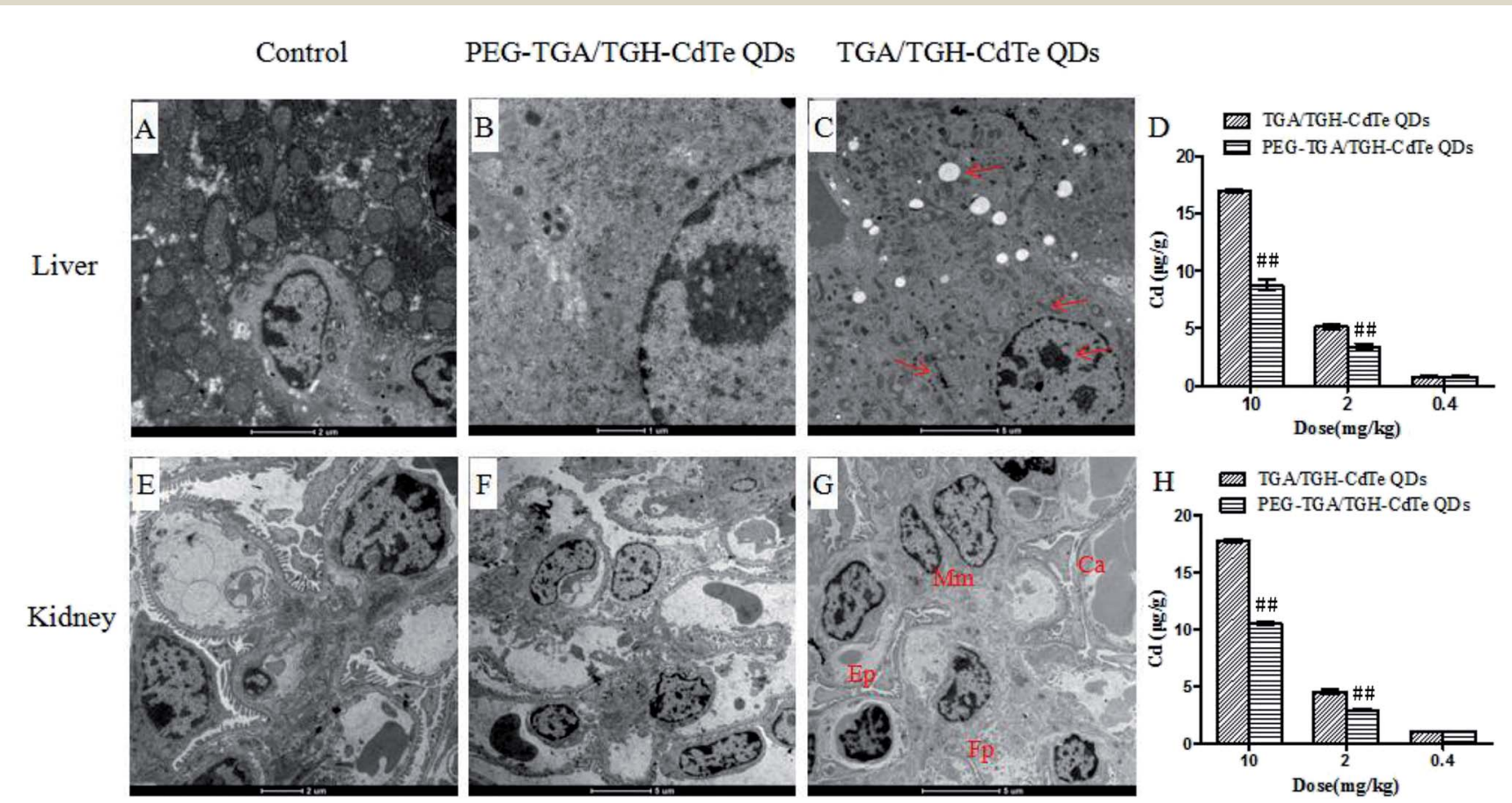

Fig. 6 Ultramicrostructure and element mapping of the liver and kidney treated with TGA/TGH-CdTe and PEG-TGA/TGH-CdTe QDs. \#\# $p<$ 0.01 vs. TGA/TGH CdTe group. 
Table 1 Mini chemistry panel test ${ }^{a}$

\begin{tabular}{|c|c|c|c|c|c|c|c|}
\hline & \multirow[b]{2}{*}{ Control } & \multicolumn{3}{|c|}{ PEG-TGA/TGH-CdTe QDs group (mg kg $\left.{ }^{-1}\right)$} & \multicolumn{3}{|c|}{ TGA/TGH-CdTe QDs group (mg kg-1) } \\
\hline & & 0.4 & 2.0 & 10 & 0.4 & 2.0 & 10 \\
\hline $\operatorname{ALT} / \mathrm{GPT}\left(\mathrm{U} \mathrm{L}^{-1}\right)$ & $36.2 \pm 2.7$ & $38.4 \pm 4.6^{\#}$ & $39.5 \pm 2.9^{\# \#}$ & $39.6 \pm 2.5$ & $34.8 \pm 3.1$ & $33.5 \pm 3.0$ & $41.7 \pm 6.2^{* *}$ \\
\hline AKP (Kings/100 mL) & $25.4 \pm 5.7$ & $24.1 \pm 5.6$ & $24.2 \pm 4.2$ & $26.6 \pm 6.8$ & $22.2 \pm 2.9$ & $25.8 \pm 3.1$ & $23.9 \pm 4.1$ \\
\hline $\operatorname{SCr}\left(\mu \mathrm{mol} \mathrm{L}^{-1}\right)$ & $50.9 \pm 7.2$ & $49.8 \pm 6.43$ & $50.0 \pm 8.6$ & $50.9 \pm 6.2^{\#}$ & $50.8 \pm 9.0$ & $54.8 \pm 7.5$ & $49.8 \pm 7.0^{*}$ \\
\hline
\end{tabular}

Table 2 Liver oxidation index ${ }^{a}$

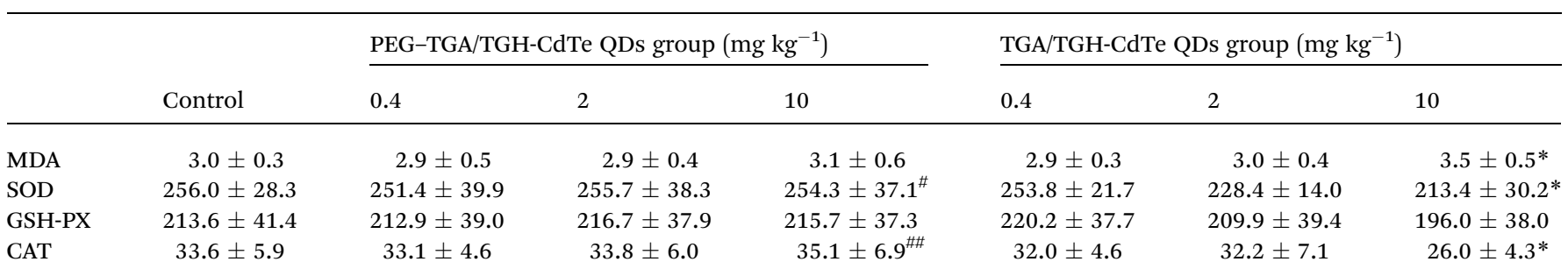

${ }^{a}$ Results are illustrated as the mean and standard deviation. ${ }^{*} p<0.05$ vs. control group; ${ }^{\#} p<0.05 v s$. TGA/TGH CdTe group; ${ }^{\# \#} p<0.01$ vs. TGA/TGH CdTe group.

Table 3 Kidney oxidation index ${ }^{a}$

\begin{tabular}{|c|c|c|c|c|c|c|c|}
\hline & \multirow[b]{2}{*}{ Control } & \multicolumn{3}{|c|}{ PEG-TGA/TGH-CdTe QDs group (mg kg $\left.{ }^{-1}\right)$} & \multicolumn{3}{|c|}{ TGA/TGH-CdTe QDs group (mg kg $\left.{ }^{-1}\right)$} \\
\hline & & 0.4 & 2 & 10 & 0.4 & 2 & 10 \\
\hline SOD & $151.9 \pm 18.4$ & $146.0 \pm 9.5$ & $149.0 \pm 8.6$ & $150.1 \pm 9.3^{\#}$ & $146.4 \pm 9.4$ & $138.8 \pm 6.8$ & $132.3 \pm 18.1^{*}$ \\
\hline GSH-PX & $245.1 \pm 42.6$ & $250.8 \pm 33.5$ & $254.7 \pm 45.3$ & $260.2 \pm 49.4$ & $262.6 \pm 43.0$ & $253.8 \pm 34.5$ & $243.6 \pm 50.0$ \\
\hline CAT & $45.8 \pm 6.3$ & $43.6 \pm 8.1$ & $45.0 \pm 7.1$ & $43.3 \pm 8.6$ & $42.7 \pm 7.8$ & $43.7 \pm 7.3$ & $39.9 \pm 8.2$ \\
\hline
\end{tabular}

${ }^{a}$ Results are illustrated as the mean and standard deviation. ${ }^{*} p<0.05$ vs. control group; ${ }^{\#} p<0.05$ vs. TGA/TGH CdTe group.

previous studies have not provided reproducible results to consider that QDs have prominent toxicity in animals at physiologically appropriate doses; in this study, we have discussed the mechanisms causing the difference in the toxicity before and after transformation. Oxidative stress is one of the most important mechanisms of toxicity or damage resulting from exogenous substances. Oxidative stress occurs when cells fail to balance the compensation for it, which finally destroys normal physiological redox-regulated functions and causes damage in tissue. ${ }^{29}$ The levels of MDA and the activities of SOD, GSH-PX and CAT were determined to evaluate oxidative stress in the liver and kidney with respect to the level of lesion. As shown in Table 2, the levels of MDA increased, and the activity of SOD and CAT decreased significantly in the liver in the group treated with TGA/TGH-CdTe QDs at high concentrations when compared with the case of the control group. In contrast, the quantity of these indicators had no apparent variation after exposure to the PEG-TGA/TGH CdTe QDs. Similar to the case of the liver, an increase in the MDA level and a significant decline in the SOD activity were observed in the kidney in the high-dose TGA/TGHCdTe QD-treated group when compared with the case of the control group (Table 3). However, the activity of CAT dropped without significant difference. On the other hand, there was no dramatic alteration in the mice after the PEG-TGA/TGH CdTe QD treatment. Based on the abovementioned results, both the accumulation of cadmium and oxidative stress instead of an isolation factor were responsible for the in vivo toxicity of these QDs. Thus, PEG played a role in both decreasing the accumulation and changing the oxidative stress, which contributed to the higher biocompatibility of the QDs.

\section{Conclusion}

In this study, we performed a systematic investigation to compare the acute toxicity in mice models treated with TGA/ TGH CdTe and PEG-TGA/TGH CdTe QDs. The in vitro and in vivo toxicity were measured and compared to illustrate the change after the surface of the QDs was modified by PEG. The 
results obtained from the investigation of two probable mechanisms indicated that free cadmium ions and variation in the oxidative stress parameters act together to cause the toxicity of TGA/TGH-CdTe QDs. PEG modification played an important role in reducing the toxicity of the QDs. PEG conjugated with the QD surface through chemical bonds and thus altered their surface state. Furthermore, PEG formed a fence-like structure on the QD surface, which could more effectively prevent $\mathrm{Cd}^{2+}$ release, which was induced by the diffusion effect from the QD surface to the solution. This study provides invaluable information to enhance the safety and explain the toxicity of QDs. Note that various factors, such as composition, surface modification agents, size and dose, play a role in the toxicity of QDs; thus, some novel approaches need be introduced to achieve a comprehensive understanding of them.

\section{Conflicts of interest}

There are no conflicts to declare.

\section{Acknowledgements}

This work was supported by the Natural Science Foundation of Jiangsu Province, China (BK20161173) and Postgraduate Research \& Practice Innovation Program of Jiangsu Province, China (KYCX18_2200) and Xuzhou Natural Science Foundation, China (KC18201 and KC18108).

\section{References}

1 M. Chu, X. Pan, D. Zhang, Q. Wu, J. Peng and W. Hai, Biomaterials, 2012, 33, 7071-7083.

2 Y. Yu, L. Xu, J. Chen, H. Gao, S. Wang, J. Fang and S. Xu, Colloids Surf., B, 2012, 95, 247-253.

3 X. Xiang, T. Gao, B. R. Zhang, F. L. Jiang and Y. Liu, Toxicol. Res., 2018, 7, 1071-1080.

4 P. Meng, Y. Xiong, Y. Wu, Y. Hu, H. Wang, Y. Pang, S. Jiang, S. Han and P. Huang, Chem. Commun., 2018, 54, 5342-5345.

5 H. Buchtelova, V. Strmiska, Z. Skubalova, S. Dostalova, P. Michalek, S. Krizkova, D. Hynek, L. Kalina, L. Richtera, A. Moulick, V. Adam and Z. Heger, J. Nanobiotechnol., 2018, 16, 43.

6 J. Wang, H. Sun, P. Meng, M. Wang, M. Tian, Y. Xiong, X. Zhang and P. Huang, Int. J. Nanomed., 2017, 12, 64256435.

7 T. Zhang, Y. Hu, M. Tang, L. Kong, J. Ying, T. Wu, Y. Xue and Y. Pu, Int. J. Mol. Sci., 2015, 16, 23279-23299.

8 X. J. Liu, P. J. Zhou, H. J. Zhan, H. Y. Liu, J. W. Zhang and Y. N. Zhao, RSC Adv., 2017, 7, 29998-30007.
9 J. Chen, X. Q. Yang, M. Y. Qin, X. S. Zhang, Y. Xuan and Y. D. Zhao, J. Nanobiotechnol., 2015, 13, 76.

10 T. Aubert, S. J. Soenen, D. Wassmuth, M. Cirillo, R. Deun Van, K. Braeckmans and Z. Hens, ACS Appl. Mater. Interfaces, 2014, 6, 11714-11723.

11 H. Chen, T. Wang, K. Li, Q. He, X. Hou, R. Yang and B. Wang, J. Colloid Interface Sci., 2017, 485, 51-58.

12 N. Chen, Y. He, Y. Su, X. Li, Q. Huang, H. Wang, X. Zhang, R. Tai and C. Fan, Biomaterials, 2012, 33, 1238-1244.

13 R. Zhang, X. Zhang, S. Gao and R. Liu, Sci. Total Environ., 2018, 655, 1334-1341.

14 A. Yamindago, N. Lee, S. Woo, H. Choi, J. Y. Mun, S. W. Jang, S. I. Yang, F. Anton-Erxleben, T. C. G. Bosch and S. Yum, Aquat. Toxicol., 2018, 205, 130-139.

15 K. Shanker, J. Naradala, G. K. Mohan, G. S. Kumar and P. L. Pravallika, RSC Adv., 2017, 7, 37158-37167.

16 W. Zhang, L. Yang, H. Kuang, P. Yang, Z. P. Aguilar, A. Wang, F. Fu and H. Xu, J. Hazard. Mater., 2016, 318, 61-69.

17 J. Song, Z. Dai, W. Guo, Y. Li, W. Wang, N. Li and J. Wei, J. Nanosci. Nanotechnol., 2013, 13, 6924-6927.

18 A. Sadaf, B. Zeshan, Z. Wang, R. Zhang, S. Xu, C. Wang and Y. Cui, J. Nanosci. Nanotechnol., 2012, 12, 8287-8292.

19 Z. Zhao, Y. Wang, J. Han, K. Wang, D. Yang, Y. Yang, Q. Du, Y. Song and X. Yin, Int. J. Nanomed., 2014, 9, 5849-5862.

20 R. Mare, D. Paolino, C. Celia, R. Molinaro, M. Fresta and D. Cosco, Int. J. Pharm., 2018, 552, 414-421.

21 X. Tang, J. Sun, T. Ge, K. Zhang, Q. Gui, S. Zhang and W. Chen, Colloids Surf., B, 2018, 172, 26-36.

22 Y. Du, D. Yang, S. Sun, Z. Zhao and D. Tang, Luminescence, 2015, 30, 519-525.

23 J. M. Harris, E. C. Struck, M. G. Case, M. S. Paley, J. M. Vanalstine and D. E. Brookes, J. Polym. Sci., Part A: Polym. Chem., 1984, 22, 341-352.

24 National Research Council, Guide for the Care and Use of Laboratory Animals, The National Academies Press, Washington, DC, 8th edn, 2011.

25 Y. Lu, Y. Su, Y. Zhou, J. Wang, F. Peng, Y. Zhong, Q. Huang, C. Fan and Y. He, Biomaterials, 2013, 34, 4302-4308.

26 A. M. Derfus, W. C. W. Chan and S. N. Bhatia, Nano Lett., 2004, 4, 11-18.

27 B. I. Ipe, M. Lehnig and C. M. Niemeyer, Small, 2005, 1, 706709.

28 K. M. Tsoi, Q. Dai, B. A. Alman and W. C. W. Chan, Acc. Chem. Res., 2013, 46, 662-671.

29 F. M. Winnik and D. Maysinger, Acc. Chem. Res., 2013, 46, 672-680. 